\title{
Assessment of Macular Function by Multifocal Electroretinogram in Patients with Multiple Sclerosis Treated with Fingolimod
}

\author{
Lucilla Barbano · Lucia Ziccardi · Doriana Landi · Carolina Gabri Nicoletti • \\ Giorgia Mataluni · Benedetto Falsini · Diego Centonze • \\ Girolama Alessandra Marfia · Luciano Quaranta · Vincenzo Parisi
}

Received: March 1, 2021 / Accepted: March 24, 2021 / Published online: June 9, 2021

(C) The Author(s) 2021

\section{ABSTRACT}

Introduction: This study aimed to evaluate whether treatment with fingolimod (FTY) may induce functional changes on the macular preganglionic retinal elements in patients affected by relapsing-remitting multiple sclerosis (RRMS) without optic neuritis (ON).

Methods: This case-control observational and retrospective study assessed multifocal electroretinogram (mfERG) responses from 35

Lucilla Barbano and Lucia Ziccardi equal contribution.

L. Barbano - L. Ziccardi · V. Parisi

IRCCS Fondazione Bietti, Rome, Italy

D. Landi · C. G. Nicoletti · G. Mataluni ·

D. Centonze - G. A. Marfia

Multiple Sclerosis Clinical and Research Unit,

Department of Systems Medicine, Tor Vergata

University, Via Montpellier 1, 00133 Rome, Italy

B. Falsini

Ophthalmology Department, IRCCS Fondazione

Policlinico Universitario A. Gemelli, Catholic

University, Rome, Italy

D. Centonze - G. A. Marfia

Unit of Neurology, IRCCS Neuromed, Via Atinense

18, 86077 Pozzilli, IS, Italy

L. Quaranta $(\bowtie)$

Department of Surgical and Clinical, Diagnostic and Pediatric Sciences, Section of Ophthalmology,

University of Pavia, IRCCS Fondazione Policlinico

San Matteo, Pavia, Italy

e-mail: luciano.quaranta@unipv.it healthy controls (mean age $43.58 \pm 5.76$ years), 41 patients with RR-MS without ON (mean age $40.64 \pm 4.83$ years, MS-noFTY group), and from 21 patients with RR-MS without ON (mean age $42.38 \pm 12.34$ years) and treated with fingolimod (Gilenya ${ }^{\circledR}$, Novartis Europharm, $0.5 \mathrm{mg} /$ day) (MS-FTY group). MfERG N1 and P1 implicit times (ITs), and N1-P1 response amplitude densities (RADs) were measured from concentric rings (R) with increasing foveal eccentricity: $0-5^{\circ}$ (R1), 5-10 ${ }^{\circ}$ (R2), $10-15^{\circ}$ (R3), $15-20^{\circ}$ (R4), $20-25^{\circ}$ (R5). We considered R1 and $\mathrm{R} 2$ as "central macular areas" and R3, R4 and R5 as "more eccentric retinal areas". In the MS-FTY group, mfERG recordings were performed between 6 and 12 months (mean $7.2 \pm 1.5$ months) from the start of FTY.

Results: In the MS-FTY group, the mean values of mfERG N1 and P1 ITs and RADs detected in both central macular areas (R1 and R2) and in more eccentric retinal areas (R3, R4 and R5) were not significantly different $(p>0.01)$ with respect to those of control and MS-noFTY groups.

Conclusions: Our mfERG results suggest that the chronic use of FTY does not induce a dysfunction of pre-ganglionic retinal elements located in the $0-25^{\circ}$ of central retina. Since FTY does not cause any retinal functional abnormality, we suggest that FTY treatment could not produce any toxic effect on pre-ganglionic retinal elements even in the absence of macular oedema. 
Keywords: Multiple sclerosis; Fingolimod; Retinal function; Outer retina; Multifocal electroretinogram

\section{Key Summary Points}

Why carry out this study?

Fingolimod is an orally administered drug used for the treatment of multiple sclerosis through its immunomodulating mechanism by binding sphingosine-1phosphate receptors on lymphocytes.

The effects of fingolimod on the sphingosine-1-phosphate receptors of the retina are not well understood.

This work aimed to study the function of the pre-ganglionic elements, located on the $0-25^{\circ}$ of the central retina, by multifocal electroretinogram recordings, in patients affected by multiple sclerosis treated with fingolimod, in order to investigate the potential treatmentrelated retinal dysfunction.

\section{What was learned from the study?}

In patients with multiple sclerosis treated with fingolimod, normal function of preganglionic elements was observed, thus suggesting that the long-term administration of fingolimod does not functionally affect photoreceptors and bipolar cells.

Multifocal electroretinogram is a useful tool able to investigate the potential treatment-related retinal toxicity, adding information to the common optical coherence tomography evaluation done during fingolimod administration in patients with multiple sclerosis.

\section{DIGITAL FEATURES}

This article is published with digital features, including a summary slide, to facilitate understanding of the article. To view digital features for this article go to https://doi.org/10.6084/ m9.figshare.14270042.

\section{INTRODUCTION}

Fingolimod (FTY) is the first orally administered drug approved in 2010 by the US Food and Drug Administration for the treatment of relapsing-remitting multiple sclerosis (RR-MS) [1].

Several clinical trials demonstrated the efficacy of FTY in reducing the frequency of relapses and the disability progression in patients with MS when compared to placebo [2, 3]. Its beneficial therapeutic effect in MS is due to the capacity of sequestrating lymphocytes into lymph nodes by binding to the sphingosine-1phosphate (S1P) receptors $[4,5]$. These receptors are expressed not only by lymphocytes but also by many other non-immune cells and they play an important role in the physiology of the cardiovascular and nervous systems and of the eye $[6,7]$. Therefore, it is not surprisingly that these organs represent privileged sites of potential drug adverse effects [5].

In the eye the most frequent adverse effect is the so-called fingolimod-associated macular oedema (FAME), although several other adverse effects have been reported [8-12]. Incidence of FAME ranges from 0 to $2.08 \%$; it occurs mainly during the first 3-4 months of therapy [13] and resolves spontaneously in the majority of patients after cessation of treatment. Oral administration of acetazolamide [14], non-steroidal anti-inflammatory drops [15] and intravitreal injection of steroid [16] have been also successfully used to treat macular oedema avoiding discontinuation of FTY [15-17].

In the retina, S1P receptor acts on endothelial cells to regulate barrier integrity, vascular permeability and tissue perfusion [18]; thus, FTY treatment may induce the downregulation of adhesion complexes and subsequently increase retinal vascular permeability. This may result in FAME as well as other similar vascular 
retinopathies $[5,19,20]$ frequently associated with visual impairment. The most sensitive technique for detecting these abnormalities is optical coherence tomography (OCT) [5]. However, more recently, Ziccardi et al. [20] also used OCT angiography (OCTA), to investigate choriocapillaris integrity in a patient with MS treated with FTY and affected by relapsed central serous chorioretinopathy (CSCR). Ziccardi et al.'s [20] findings suggested that during FTY treatment, transitory choroidal hyperpermeability with no permanent ischaemic changes occurs, and therefore drug discontinuation can be avoided.

Although the chronic use of FTY induces imbalances of the homeostasis of vascular permeability [21, 22], it could be interesting to study also the macular function of treated patients in the absence of morphological abnormalities (detected by OCT evaluation), to reveal possible toxic macular effects, as already described for different prolonged treatments (see hydroxychloroquine, tamoxifen, ethambutol) $[23,24]$.

Multifocal electroretinogram (mfERG) is considered the main tool able to objectively assess macular function [25], since it provides a topographical map of bioelectric responses derived from localized retinal areas, which are driven largely by the cone-related pre-ganglionic retinal components. A 'kernel analysis' applied to mfERG responses can be used to assess nonlinear functions of the visual system mainly originating from selected population of pre-ganglionic (photoreceptors and bipolar cells) elements $[26,27]$. Recently [28], it was reported that in patients with RR-MS, in the absence of optic neuritis (ON) or when after an episode of $\mathrm{ON}$ a complete recovery of visual acuity is reached, there is an absence of conerelated pre-ganglionic component dysfunction and this was detectable by mfERG responses that were not significantly different when compared to normal control ones.

The aim of the present study was to evaluate the function of cone-related pre-ganglionic components located in the macular area (0-10 central retinal degrees) and also in more eccentric retinal areas with respect to the fovea (10-25 central retinal degrees) by mfERG recordings in patients affected by RR-MS and chronically treated with FTY without ON. Our data can provide information on whether the treatment with FTY could produce toxic effects on pre-ganglionic retinal elements inducing a possible dysfunctional condition also in the absence of macular oedema detectable by OCT evaluation.

\section{METHODS}

\section{Participants}

In this observational retrospective study, 21 patients with RR-MS (mean age $42.38 \pm 12.34$ years, 14 women and 7 men, MSFTY group) were selected from a bigger cohort of 95 patients referred by the MS Clinical and Research Unit of the University of Tor Vergata of Rome for a periodic ophthalmic evaluation and macular screening for management of FTY treatment. Patients underwent a complete ophthalmological evaluation at the Visual Neurophysiology and Neurophthalmology Research Unit, IRCCS Fondazione Bietti, between September 2018 and December 2020.

These patients were selected on the basis of the following inclusion criteria:

1. Diagnosis of RR-MS according to validated 2017 Thompson criteria [29]

2. Continuative treatment with FTY for a period between 8 and 12 months

3. High-contrast best-corrected visual acuity (BCVA) of 0.0 (mean $0.0 \pm 0.0 \log$ MAR)

4. Refractive error (when present) between 3.000 and +3.000 spherical equivalent

5. Intraocular pressure less than $18 \mathrm{mmHg}$

6. Absence of history of unilateral or bilateral ON (i.e. painless reduction of BCVA, contrast sensitivity, colour vision and any type of visual field defects)

7. Absence of previous and present signs of morphological macular abnormalities studied by OCT scans (i.e. FAME, CSCR, inflammatory macular oedema) and any retinal diseases interfering with macular function

8. Absence of history of drug intake that can interfere with normal macula function 
Excluded from the present study were all patients with history of cystoid macular oedema, glaucoma, uveitis or other systemic diseases that may influence the retinal function.

On the basis of the aforementioned inclusion and exclusion criteria, we selected 35 MSFTY eyes, 24 eyes from 12 patients (both eyes included) and nine eyes from nine patients (one eye included).

A group of selected 41 age-matched RR-MS eyes of 41 patients (mean age $40.64 \pm 4.83$ years, 26 women and 15 men) subject to the same inclusion and exclusion mentioned criteria used for the MS-FTY group, but not treated with FTY were also considered (MS-noFTY group).

A group of selected 35 age-matched healthy subjects (mean age $43.58 \pm 5.76$ years, 20 women and 15 men), providing 35 healthy eyes, with BCVA of 0.0 logMAR (mean $0.0 \pm 0.0$ ), served as controls (C).

In all patients with MS and controls, the BCVA evaluation, the macular OCT study and the mfERG recordings were assessed in the same session during the same day of the examination.

In patients of the MS-FTY group, all evaluations were performed between 6 and 12 months (mean $7.20 \pm 1.50$ months) from the start of FTY (Gilenya ${ }^{\circledR}$, Novartis Europharm, tablet containing $0.5 \mathrm{mg}$ of fingolimod/day).

All procedures performed in this study were in accordance with the ethical standards of the institutional and/or national research committee and with the 1964 Helsinki declaration and its later amendments or comparable ethical standards. The study was approved by the local ethics committee (Comitato Etico Centrale IRCCS Lazio, Sezione IFO/Fondazione Bietti, Rome, Italy). Informed consent was obtained from all individual participants included in the study.

\section{Multifocal Electroretinogram Recording}

The mfERG was recorded by using a modified version of the Espion system (Diagnosys UK Ltd; Histon, Cambridge, UK) following the 2011 International Society for Clinical
Electrophysiology of Vision (ISCEV) standards [25] and by using the same previously published method [28].

Briefly, the multifocal stimulus, consisting of 61 scaled hexagons, was displayed on a highresolution, black-and-white $32^{\prime \prime}$ LCD monitor with a frame rate of $75 \mathrm{~Hz}$. The array of hexagons subtended $50^{\circ}$ of visual field $\left(25^{\circ}\right.$ radius from the fixation point to the edge of the display). Each hexagon was independently alternated between black $\left(1 \mathrm{~cd} / \mathrm{m}^{2}\right)$ and white $\left(200 \mathrm{~cd} / \mathrm{m}^{2}\right)$ according to a binary $m$-sequence. This resulted in a contrast of $99 \%$. The luminance of the monitor screen and the central fixation cross (used as target) was $100 \mathrm{~cd} / \mathrm{m}^{2}$. The visual stimulation was performed by correcting BCVA for the distance of the visual stimuli. The $m$-sequence had $2^{13-1}$ elements, and total recording time was approximately 8 min, divided into 16 segments. Between segments, the subject was allowed to rest for a few seconds. Focusing lenses were used when necessary. To maintain a stable fixation, a small red cross target $\left(0.5^{\circ}\right)$ was placed in the centre of the stimulation field. For every mfERG, the subject reported that they could clearly perceive the fixation target. The eye's position was continuously monitored by an in-built video system to track fixation losses.

MfERGs were binocularly recorded in the presence of pupils that were maximally pharmacologically dilated with $1 \%$ tropicamide to a diameter of 7-8 $\mathrm{mm}$. Pupil diameter was measured by an observer (LB) by means of a millimetre ruler and a magnifying lens and stored for each tested eye. The cornea was anaesthetized with benoxinate eye drops $0.4 \%$. MfERGs were recorded between an active Dawson-Trick-Litzkow (DTL) contact electrode and a reference electrode $(\mathrm{Ag} / \mathrm{AgCl}$ skin electrode placed on the correspondent outer canthi). A small $\mathrm{Ag} / \mathrm{AgCl}$ skin ground electrode was placed at the centre of the forehead. Interelectrode resistance was less than $3 \mathrm{k} \Omega$.

After automatic rejection of artefacts and post-acquisition processing done by the in-built Espion software, the first-order kernel response was examined. MfERG responses with a signal to noise $\geq 3$ were accepted for the analysis. 
In the analysis of mfERG responses, we considered, for each obtained averaged response, the implicit times (ITs) of the first negative peak (N1) and the first positive peak (P1) measured in milliseconds and the N1-P1 peak-to-peak response amplitude density (RAD) measured in nanovolt/degree ${ }^{2}$.

We considered the averaged response obtained from five concentric annular retinal areas (rings) centred on the fovea: $0-5^{\circ}$ (ring 1 ,

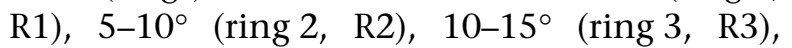
$15-20^{\circ}$ (ring $\left.4, \mathrm{R} 4\right)$ and $20-25^{\circ}$ (ring 5, R5). We considered R1 and R2 as "central macular areas" and R3, R4 and R5 as "more eccentric retinal areas".

\section{Statistical Analysis}

The number of subjects analysed in this study was derived from mfERG data previously acquired and used [29]. Therefore, considering the mean R1 amplitude value of mfERG descriptive statistic equal to $50.1 \mathrm{nV} /$ degree $^{2}$, standard deviation $(\mathrm{SD})=8.3 \mathrm{nV} /$ degree $^{2}$ for controls, mean R1 amplitude equal to $41.0 \mathrm{nV} /$ degree $^{2}, \mathrm{SD}=10.4 \mathrm{nV} /$ degree $^{2}$ for each patient group, we obtained 35 observations in both groups with $\alpha=0.01$ and power $=0.90$.

The differences of mfERG N1 and P1 IT and N1-P1 RAD mean values from each ring on the retinal map (from ring 1 to ring 5), between controls, MS-noFTY and MS-FTY groups, were analysed by one-way analysis of variance (ANOVA). $P$ values lower than 0.01 were considered as statistically significant, corrected for multiple comparison $(N=3, p=0.05 / N)$ using the Bonferroni test.

Minitab 17 (version 1) software was used for statistical analysis.

\section{RESULTS}

Figure 1 presents representative mfERG averaged traces obtained in eyes from a control, a MS-FTY and a MS-noFTY patient by using ring analysis.

Table 1 reports the mfERG N1 and P1 IT and N1-P1 RAD mean values from each ring (R1 to
R5) recorded in control, MS-FTY and MS-noFTY groups and relative statistics between groups.

\section{Evaluation of Central Macular Areas $\left(0-10^{\circ}\right)$}

When the mfERG responses obtained in rings 1 and 2 are considered, in the MS-FTY group, N1IT, P1-IT and RAD values were not significantly different when compared with those of the control group [ $f(1,69) ; p>0.01]$ and those of the MS-noFTY group $[f(1,75) ; p>0.01]$.

\section{Evaluation of More Eccentric Retinal Areas $\left(10-25^{\circ}\right)$}

When the mfERG responses obtained in rings 3, 4 and 5 are considered, N1-IT, P1-IT and RAD values detected in the MS-FTY group were not significantly different when compared with those of the control group [f $(1,69) ; p>0.01]$ and those of the MS-noFTY group $[f(1,75)$; $p>0.01]$.

\section{DISCUSSION}

The aim of the present study was to evaluate the function of the pre-ganglionic elements (photoreceptors and bipolar cells), located in the central retina, by mfERG in patients affected by RR-MS and chronically treated with FTY, to verify possible drug toxicity. To compare data between homogenous groups, we studied also eyes from patients with MS not treated with FTY and from age-matched healthy controls.

On the basis of our recent findings [28], patients with MS with ON and poor recovery of BCVA as well as with FAME and with any other retina and systemic conditions that are known to affect macular function were excluded. Indeed, on the basis of the possibility that macular function may be affected by the event of $\mathrm{ON}$, in a recent work we reported that MS eyes with $\mathrm{ON}$ and poor recovery of BCVA showed reduced mfERG RADs in ring 1 [29]. By contrast, other studies $[29,30]$ in patients with MS with or without history of $\mathrm{ON}$ and full recovery of BCVA reported the absence of 


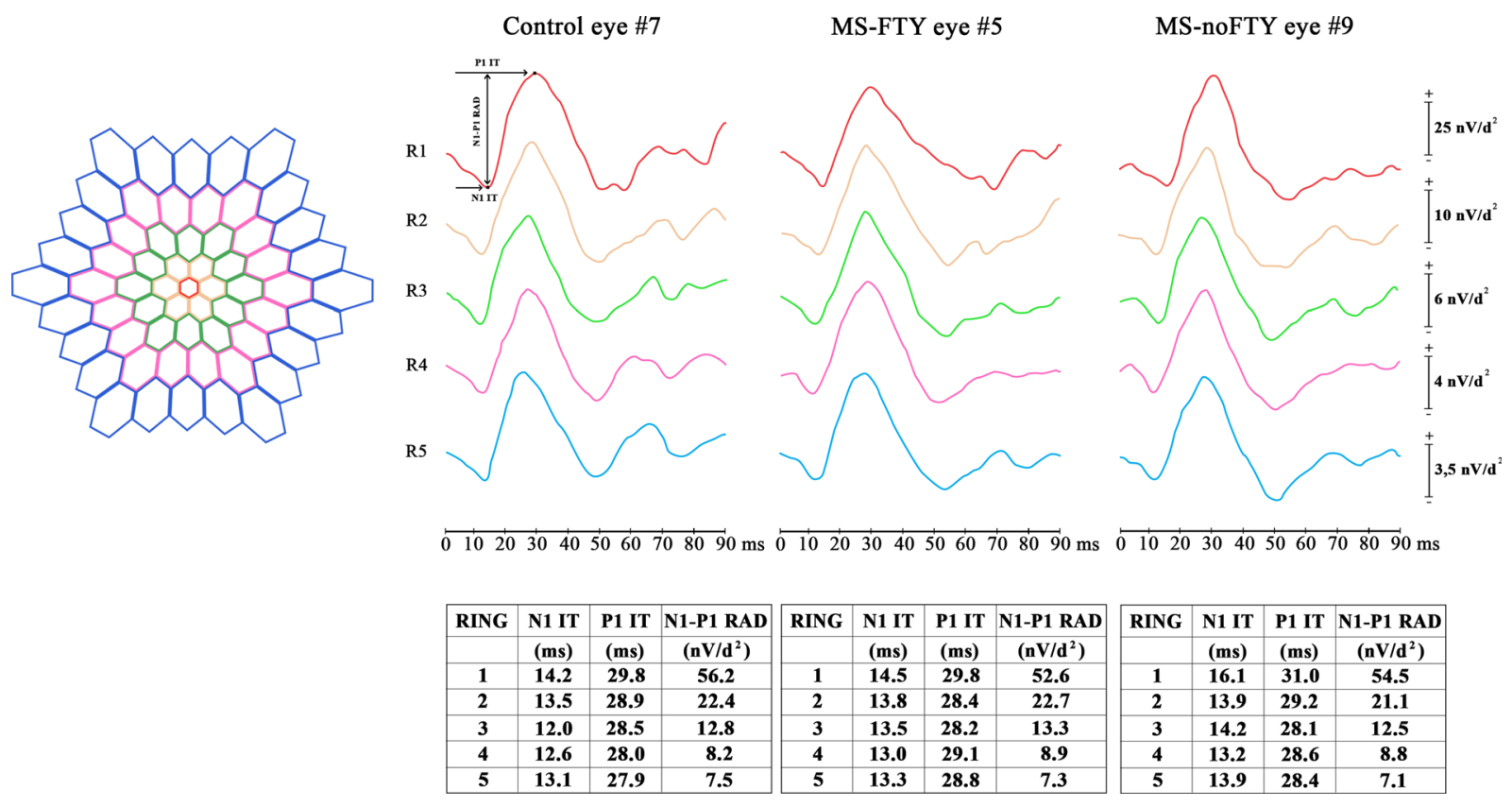

Fig. 1 Multifocal electroretinogram (mfERG) averaged traces obtained in eyes from a control patient (\#7), a patient with multiple sclerosis treated with fingolimod (MS-FTY\#5), and a patient not treated with fingolimod (MS-noFTY\#9) by using ring analysis. The averaged values of $\mathrm{N} 1$ and P1 implicit times (IT, measured in milliseconds) and of N1-P1 response amplitude density (RAD,

mfERG abnormalities detectable on the central fovea and on more eccentric retinal areas with respect to the fovea. All this is relevant for the possibility to detect true variations of mfERG parameters in the MS-FTY cohort of the present work. On the basis of the possibility that FAME also could induce mfERG abnormalities leading to bias for our data, we also excluded patients with exudative maculopathies, such as subjects with diabetes [31] or patients with retinal vein occlusion [32] that have been reported to show reduced amplitudes and/or implicit time delay of mfERG.

By comparing the values of mfERG parameters between the studied groups, we found nonstatistically significant differences in function of both central macular area (0-10 central degrees) and more eccentric retinal areas $\left(10-25^{\circ}\right)$ in MSFTY eyes when compared to MS-noFTY and control groups. This suggests that by means of an objective method (mfERG recordings) we were able to identify that retinal pre-ganglionic measured in nanovolt $/$ degree $^{2}$ ) were obtained from five concentric annular retinal regions (rings) centred on the fovea: $0-5^{\circ}$ (ring 1, R1), 5-10 ${ }^{\circ}$ (ring 2, R2), 10-15 (ring 3, R3), $15-20^{\circ}$ (ring 4, R4) and $20-25^{\circ}(\operatorname{ring} 5, \mathrm{R} 5)$. On the left, the topographical map of scaled hexagons covering $25^{\circ}$ of mfERG ring analysis is shown

elements are not functionally impaired by FTY treatment, even when macular oedema is not clinically detectable (by ophthalmoscopy or OCT evaluation).

Therefore, we objectively excluded potential retinal damage of photoreceptors and bipolar cells induced by FTY in patients with MS. The presence of a normal retinal function in MS-FTY eyes may exclude toxic damage on retinal cells. On the contrary, other treatments (such as chloroquine and hydroxychloroquine [23, 24]) are known to impair retinal pigmented epithelium and secondarily photoreceptors, with a risk of toxicity dependent on the dose and on the duration of intake. Indeed, to reveal the risk of toxic maculopathy induced by these substances, mfERG remains the gold standard in clinical settings [24].

To our knowledge, the hypothesis that FTY might have a protective role in the retina [33] should be also considered. In fact, it is known that the use of substances that modulate S1P 


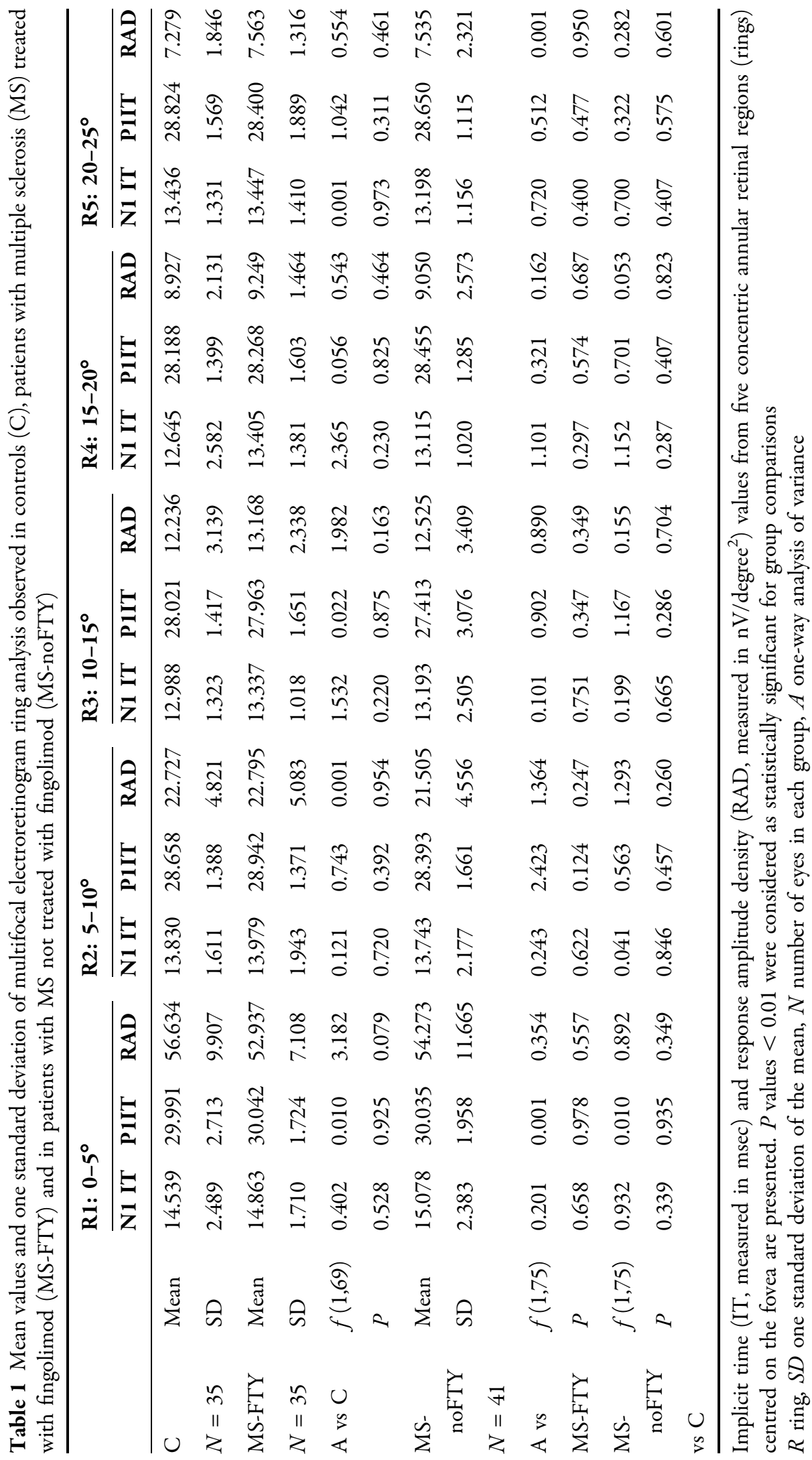


signalling, such as FTY, preserves neuronal viability and retinal function [33, 34]. In the animal retina, S1P induces the proliferation and later differentiation of retinal progenitors into photoreceptors and mediates photoreceptor survival upon oxidative damage [35]. Sphingolipids are known to modulate the functionality of multiple cell types present in the retina, and the dysregulation of their metabolism may contribute to the onset and progression of retinal diseases [33, 34]. In particular, ceramide, the core lipid of sphingolipid metabolism, is a key factor in apoptotic cell death, and its increased levels are associated with death of photoreceptors and retinal pigment epithelium cells [36]. Indeed, studies in animal models described that FTY inhibits ceramide synthase and blocks de novo ceramide production, suggesting that FTY effectively prevents ceramide increase in the retina, protects photoreceptors from apoptosis and delays retinal degeneration $[37,38]$. In the end, S1P receptors play more than one role: on the one hand, they prevent degeneration of photoreceptors and ganglion cells [33-35], and on the other hand, since they are highly expressed on endothelial cells, they are implicated in the regulation of the vascular endothelial barrier and in the intercellular junctions $[39,40]$.

In addition, since it is known that photoreceptors are mainly supplied by nutrients transported through the fenestrations of the choriocapillaris [41], it is likely that these retinal elements are not functionally influenced by the vascular S1P-mediated homoeostasis, which is more relevant at the level of the retinal vessels localized between the ganglion cell and inner nuclear layers.

\section{CONCLUSION}

Since mfERG recording represents an objective method to investigate the function of pre-ganglionic elements, its use, combined to OCT evaluation, may provide reliable information about the morpho-functional condition of the central retina during FTY treatment.

Our mfERG results suggest that the chronic use of FTY does not interfere with the regular function of photoreceptors and bipolar cells, which are localized between the choriocapillaris and the retinal deep capillary plexus. Therefore, we assume that neither indirect damage nor lack of nutritional vascular supply to photoreceptors and bipolar cells is present in the course of FTY treatment. All this led us to believe that the treatment with FTY should not produce toxic effects on pre-ganglionic retinal elements, as it does not induce any dysfunction also in the absence of macular oedema, detectable by OCT evaluation.

Nevertheless, a limitation of this work is that the observations were made in patients with MS within an interval of 6-12 months (mean $7.20 \pm 1.50$ months) from the treatment start. Therefore our data should be confirmed using a longer follow-up time to investigate potential long-term dysfunctional effects of FTY on preganglionic retinal elements.

Further studies are needed to investigate the correlations between retinal vasculature features (by OCT-A) and retinal function (by mfERG) in MS-FTY patients, to explore the hypothesis of a potential protective role of FTY in retinal degeneration $[37,38]$.

\section{ACKNOWLEDGEMENTS}

We thank the participants of the study. This manuscript is based on our previously published work [28], reporting the multifocal electroretinogram data obtained in patients with multiple sclerosis without optic neuritis. The contribution of Bietti Foundation for this study was supported by the Italian Ministry of Health and Fondazione Roma. Authors acknowledge Dr. Federica Petrocchi for executing BCVA measurements and Dr. Maria Luisa Alessi for technical assistance.

Funding. Open access funding provided by Università degli Studi di Pavia within the CRUICARE Agreement.

Authorship. All named authors meet the International Committee of Medical Journal Editors (ICMJE) criteria for authorship for this 
article, take responsibility for the integrity of the work as a whole, and have given their approval for this version to be published.

Author Contributions. Conception and design: Lucilla Barbano, Lucia Ziccardi and Vincenzo Parisi; data collection: Lucilla Barbano, Doriana Landi, Carolina Gabri Nicoletti, Giorgia Mataluni; statistical analysis and data interpretation: Lucilla Barbano, Lucia Ziccardi, Diego Centonze, Girolama Alessandra Marfia, Benedetto Falsini, Luciano Quaranta, Vincenzo Parisi; drafting the manuscript: Lucilla Barbano, Lucia Ziccardi, Benedetto Falsini, Diego Centonze, Girolama Alessandra Marfia, Luciano Quaranta, Vincenzo Parisi; overall responsibility: Vincenzo Parisi.

Disclosures. Lucilla Barbano, Lucia Ziccardi, Doriana Landi, Carolina Gabri Nicoletti, Giorgia Mataluni, Benedetto Falsini, Diego Centonze, Girolama Alessandra Marfia, Luciano Quaranta and Vincenzo Parisi have nothing to disclose.

Compliance with Ethics Guidelines. All procedures performed in this study were in accordance with the ethical standards of the institutional and/or national research committee and with the 1964 Helsinki declaration and its later amendments or comparable ethical standards. The study was approved by the local ethics committee (Comitato Etico Centrale IRCCS Lazio, Sezione IFO/Fondazione Bietti, Rome, Italy). Informed consent was obtained from all individual participants included in the study.

Data Availability. All authors had full access to all of the data in this study and take complete responsibility for the integrity of the data and accuracy of the data analysis. The datasets generated and/or analyzed during the current study are available from the corresponding author on reasonable request.

Open Access. This article is licensed under a Creative Commons Attribution-NonCommercial 4.0 International License, which permits any non-commercial use, sharing, adaptation, distribution and reproduction in any medium or format, as long as you give appropriate credit to the original author(s) and the source, provide a link to the Creative Commons licence, and indicate if changes were made. The images or other third party material in this article are included in the article's Creative Commons licence, unless indicated otherwise in a credit line to the material. If material is not included in the article's Creative Commons licence and your intended use is not permitted by statutory regulation or exceeds the permitted use, you will need to obtain permission directly from the copyright holder. To view a copy of this licence, visit http://creativecommons.org/licenses/by$\mathrm{nc} / 4.0 /$.

\section{REFERENCES}

1. Cohen JA, Barkhof F, Comi G, et al. Oral fingolimod or intramuscular interferon for relapsing multiple sclerosis. N Engl J Med. 2010;362:402-15.

2. Kappos L, Radue E-W, O'Connor P, et al. A placebocontrolled trial of oral fingolimod in relapsing multiple sclerosis. N Engl J Med. 2010;362:387-401.

3. Kappos L, O'Connor P, Radue EW, et al. Long-term effects of fingolimod in multiple sclerosis: the randomized FREEDOMS extension trial. Neurology. 2015;84:1582-91.

4. Chun J, Hartung H. Mechanism of action of oral fingolimod (FTY720) in multiple sclerosis. Clin Neuropharmacol. 2010;33:91-101.

5. Mandal P, Gupta A, Fusi-Rubiano W, Keane PA, Yang Y. Fingolimod: therapeutic mechanisms and ocular adverse effects. Eye (Lond). 2017;31:232-40.

6. Hla T. Physiological and pathological actions of sphingosine 1-phosphate. Semin Cell Dev Biol. 2004; 15:513-20.

7. Chaudhry BZ, Cohen JA, Conway DS. Sphingosine 1-phosphate receptor modulators for the treatment of multiple sclerosis. Neurotherapeutics. 2017;14: 859-73.

8. Christopher KL, Elner VM, Demirci H. Conjunctival lymphoma in a patient on fingolimod for relapsingremitting multiple sclerosis. Ophthal Plast Reconstr Surg. 2017;33:e73-5. 
9. Bhatti MT, Freedman SM, Mahmoud TH. Fingolimod therapy and macular hemorrhage. J Neuroophthalmol. 2013;33:370-2.

10. Sia PI, Aujla JS, Chan WO, Simon S. Fingolimodassociated retinal hemorrhages and roth spots. Retina. 2018;38:e80-1.

11. Ueda N, Saida K. Retinal hemorrhages following fingolimod treatment for multiple sclerosis; a case report. BMC Ophthalmol. 2015;15:135.

12. Abraham A, Nicholson L, Dick A, Rice C, Atan D. Intermediate uveitis associated with MS: diagnosis, clinical features, pathogenic mechanisms, and recommendations for management. Neurol Neuroimmunol Neuroinflamm. 2020;8:e909.

13. Goh LY, Kirthi V, Silber E, Harvey JP, Jackson TL. Real-world incidence of fingolimod-associated macular oedema. Mult Scler Relat Disord. 2020;42: $102-25$.

14. Schröder K, Finis D, Harmel J, et al. Acetazolamide therapy in a case of fingolimod-associated macular edema: early benefits and long-term limitations. Mult Scler Relat Disord. 2015;4:406-8.

15. Husmann R, Davies JB, Ghannam M, Berry B, Kelkar P. Fingolimod-associated macular edema controlled with nepafenac non-steroidal anti-inflammatory opthalmologic applications. Clin Mol Allergy. 2020;18:3.

16. Thoo S, Cugati S, Lee A, Chen C. Successful treatment of fingolimod-associated macular edema with intravitreal triamcinolone with continued fingolimod use. Mult Scler. 2015;21:249-51.

17. Akiyama H, Suzuki Y, Hara D, et al. Improvement of macular edema without discontinuation of fingolimod in a patient with multiple sclerosis: a case report. Medicine (Baltimore). 2016;95:e4180.

18. Lucke S, Levkau B. Endothelial functions of sphingosine-1-phosphate. Cell Physiol Biochem. 2010;26:87-96.

19. McVerry BJ, Garcia JG. Endothelial cell barrier regulation by sphingosine 1-phosphate. J Cell Biochem. 2004;92:1075-85.

20. Ziccardi L, Landi D, De Geronimo D, et al. Choriocapillaris integrity in relapsed central serous chorioretinopathy in a patient treated with fingolimod for multiple sclerosis: new insights from optical coherence tomography angiography. J Neuroophthalmol. 2021;41:e51-3.

21. Proia RL, Hla T. Emerging biology of sphingosine-1phosphate: its role in pathogenesis and therapy. J Clin Invest. 2015;125:1379-87.
22. Ocwieja M, Meiser K, David OJ, et al. Effect of fingolimod (FTY720) on cerebral blood flow, platelet function and macular thickness in healthy volunteers. Br J Clin Pharmacol. 2014;78:1354-65.

23. Marmor MF, Kellner U, Lai TY, Melles RB, Mieler WF, American Academy of Ophthalmology. Recommendations on screening for chloroquine and hydroxychloroquine retinopathy (2016 revision). Ophthalmology. 2016;123:1386-94.

24. Dettoraki M, Moschos MM. The role of multifocal electroretinography in the assessment of drug-induced retinopathy: a review of the literature. Ophthalmic Res. 2016;56:169-77.

25. Hood DC, Bach M, Brigell M, et al. ISCEV standard for clinical multifocal electroretinography (mfERG) (2011 edition) Doc Ophthalmol. 2012;124:1-13.

26. Bearse MA, Sutter EE. Imaging localized retinal dysfunction with the multifocal electroretinogram. J Opt Soc Am A Opt Image Sci Vis. 1996;13:634-40.

27. Hood DC. Assessing retinal function with the multifocal technique. Prog Retin Eye Res. 2000;19: 607-46.

28. Ziccardi L, Barbano L, Boffa L, et al. Functional assessment of outer and middle macular layers in multiple sclerosis. J Clin Med. 2020;9:3766.

29. Thompson AJ, Banwell BL, Barkhof F, et al. Diagnosis of multiple sclerosis: 2017 revisions of the McDonald criteria. Lancet Neurol. 2018;17:162-73.

30. Gundogan FC, Demirkaya S, Sobaci G. Is optical coherence tomography really a new biomarker candidate in multiple sclerosis?-A structural and functional evaluation. Investig Ophthalmol Vis Sci. 2007;48:5773-81.

31. Greenstein VC, Holopigian K, Hood DC. The nature and extent of retinal dysfunction associated with diabetic macular oedema. Invest Ophthalmol Vis Sci. 2000;41:3643-54.

32. Abdel-Kader M, El-Dessouky WM. Multifocal electroretinogram in retinal vein occlusion. Saudi J Ophthalmol. 2010;24:125-32.

33. Simon MV, Basu SK, Qaladize B, Grambergs RC, Rotstein NP, Mandal N. Sphingolipids as critical players in retinal physiology and pathology. J Lipid Res. 2020:jlr.TR120000972. https://doi.org/10. 1194/jlr.TR120000972.

34. Simón MV, Prado Spalm FH, Vera MS, Rotstein NP. Sphingolipids as emerging mediators in retina degeneration. Front Cell Neurosci. 2019;13:246. 
35. Miranda GE, Abrahan CE, Politi LE, Rotstein NP. Sphingosine-1-phosphate is a key regulator of proliferation and differentiation in retina photoreceptors. Invest Ophthalmol Vis Sci. 2009;50:4416-28.

36. Miranda GE, Abrahan CE, Agnolazza DL, Politi LE, Rotstein NP. Ceramide-1-phosphate, a new mediator of development and survival in retina photoreceptors. Invest Ophthalmol Vis Sci. 2011;52: 6580-8.

37. Chen H, Tran JT, Eckerd A, et al. Inhibition of de novo ceramide biosynthesis by FTY720 protects rat retina from light-induced degeneration. J Lipid Res. 2013;54:1616-29.

38. Stiles M, Qi H, Sun E, et al. Sphingolipid profile alters in retinal dystrophic $\mathrm{P} 23 \mathrm{H}-1$ rats and systemic FTY720 can delay retinal degeneration. J Lipid Res. 2016;57:818-31.

39. McVerry BJ, Peng X, Hassoun PM, Sammani S, Simon BA, Garcia JG. Sphingosine 1-phosphate reduces vascular leak in murine and canine models of acute lung injury. Am J Respir Crit Care Med. 2004;170:987-93.

40. Tauseef M, Kini V, Knezevic N, et al. Activation of sphingosine kinase-1 reverses the increase in lung vascular permeability through sphingosine-1-phosphate receptor signaling in endothelial cells. Circ Res. 2008;103:1164-72.

41. Lutty GA, Hasegawa T, Baba T, Grebe R, Bhutto I, McLeod DS. Development of the human choriocapillaris. Eye (Lond). 2010;24:408-15. 